Mental Health and Learning Disabilities Research and Practice, 2009, 6, 5 -19

Management of Children with Attention Deficit/Hyperactivity Disorder and Learning Disabilities:

A Survey of Paediatric Occupational Therapists in the United Kingdom

$$
\text { Georgia Spiliotopoulou }{ }^{1}
$$

${ }^{1}$ Brunel University 


\title{
Management of Children with Attention Deficit/Hyperactivity Disorder and Learning Disabilities: A Survey of Paediatric Occupational Therapists in the United Kingdom
}

\author{
Georgia Spiliotopoulou
}

\begin{abstract}
Attention deficit/hyperactivity disorder (ADHD) is a common complex neuropsychiatric disorder frequently overlapping with learning disabilities (LD) in children with average and above intelligence (Biederman et al 1991). The complex needs of these children could be better addressed in a multidisciplinary context (Foy \& Earls 2005) within which paediatric occupational therapists may have a significant role. The aim of this study was to identify the interventions used by occupational therapists in the United Kingdom with these children and explore the rationale for their use. The purpose was to provide baseline data to inform multidisciplinary team approaches for the management of these children.
\end{abstract}

A survey, based on postal questionnaires, was conducted among 100 paediatric occupational therapists in the United Kingdom. The effective rate of response was $42 \%$. Both qualitative and quantitative data were gathered and analysed.

The findings suggested that therapists tended to use more than one method/ approach in the rehabilitation of these children. The sensory integration approach and the perceptual-motor training were more popular among therapists. A variety of other approaches, used on an individual needs basis, were reportedly used. Future studies to test the effectiveness of reported practices with children with both ADHD and LD are recommended.

Key words: attention deficit/hyperactivity disorder, learning disabilities, specific learning disabilities, occupational therapy, children.

\section{Introduction}

Attention deficit/hyperactivity disorder (ADHD) is a common neuropsychiatric disorder (Grizenko et al 2006) with at least 5\% prevalence in the general childhood population in the United Kingdom (UK) (National Institute for Clinical Excellence 2000). Brown (2000) suggests that over $50 \%$ of these children meet the diagnostic criteria for additional psychiatric and developmental disorders. More specifically, Kaplan et al (2001) found that $80 \%$ of children with ADHD had at least one other disorder. The reported estimates of prevalence of learning disabilities (LD) in ADHD range from $25 \%$ to $70 \%$ (Barkley 1994, Mayes et al 2000). Estimation differences may occur due to different sampling procedures, diagnostic criteria and assessment techniques used for several studies (Biederman et al 1991)

\section{Attention Deficit/Hyperactivity Disorder}

According to the Diagnostic and Statistical Manual of Mental Disorders - Fourth edition (DSM-IV) (American Psychiatric Association (APA) 1994), ADHD has been categorised according to whether inattention, hyperactivity or both of these types of disorders are present. Although aetiology of ADHD is poorly understood, many 
agree that results from disruptive interaction between neurochemical and neuroanatomical factors affect neurological systems (Riccio et al 1993). The executive systems subserved by the prefrontal cortex, the basal ganglia and the cerebellum, and modulated by neurotransmitters, mainly dopamine and noradrenaline, have been related to attention problems (Castellanos 1997, Lazar \& Frank 1998). Executive functions refer to problem solving skills, anticipation, strategic planning, self regulation, monitoring, maintenance of focus and task completion (Barkley 2000). Rosenman (2006) suggests that not all of the executive functions are impaired in ADHD; rather this differs across cases.

\section{Learning Disabilities}

Whitaker (2006) pinpoints the confusion around the definition of 'learning disability' (LD) in the literature. A conventional United States (US) definition relies on the IQachievement discrepancy (APA 1994). DSM-IV categorises LD in four types: reading disorders, mathematic disorders, disorders in written expression, and learning disorders not otherwise specified (APA 1994). Whitaker (2006), based on the White Paper Valuing People (Department of Health 2001), reports that in the United Kingdom (UK) the term LD refers to an IQ $<70$ and additional deficits in adaptive behaviours; whereas the US definition refers to what is called in the UK 'specific learning disabilities'. In order to discuss LD, the present paper has used US literature; therefore the term LD is used according to its US definition. The National Joint Committee on Learning Disabilities [NJCLD] (2001) attributes LD to central nervous dysfunction; still, the origin of the problem is still debatable (Spreen 2001).

\section{Overlap of ADHD with LD}

Biederman et al (1991) suggest that children who present with both ADHD and LD deserve special clinical and educational attention. Literature emphasises the need for a multimodal approach within a multidisciplinary team to address the complexity of this overlapping impairment (Cavanaugh et al 1997, Foy \& Earls 2005). The paediatric occupational therapist as a member of such teams may contribute to diagnosis and rehabilitation of these children (Chu 2003) to promote occupational health and well-being. Therefore, it is important for the multidisciplinary team to understand the different interventions that occupational therapists are using with these children in order to integrate their input in a coordinated package of care, thus addressing children's needs efficiently and holistically.

A number of international papers have examined the effectiveness of the sensory integration approach (Oetter 1986, Mulligan 1996, Parush et al 1997, Dunn 1999, Mangeot et al 2001) in occupational therapy practice with children with ADHD. This approach involves sensory stimulation (vestibular, tactile and proprioceptive) in combination with adaptive responses according to the child's neurological needs (Ayres 1979). There is also some research evidence of the benefits of specific occupational therapy programmes. The 'interactive metronome' is a computerised interactive programme developed by Cassily (1996), which is based on the perceptual motor training approach. This aims to facilitate a number of underlying central nervous system processing capacities hypothesised to be involved in motor regulation, which is thought to be necessary for optimal functioning (Koomar et al 2001). The 'alert programme' is based on the cognitive approach. It uses the analogy of a car engine to explain the level of arousal and the analogy of engine tuning to explain its regulation at appropriate levels for a 
situation or task (Williams \& Shellenberger 1994). However, currently there is limited published research describing the contemporary practice of UK occupational therapists with children with ADHD (e.g. Chu \& Reynolds 2007a, 2007b). Indeed, no papers were identified describing the approaches that occupational therapists use with children who present with both ADHD and LD (as per US definition).

\section{Present Study}

This study aimed to identify what interventions UK paediatric occupational therapists use with children who present with both ADHD and LD and to explore the rationale underpinning preferred practices. It was envisaged that information about the current range of approaches in use would provide useful baseline data to inform multidisciplinary team approaches for the management of these children. Recognising professional consensus is the least reliable level of evidence to inform evidence-based practice; nevertheless, understanding practice is a useful platform on which to build effective interventions for the targeted children.

\section{Method}

\section{Design}

This was a cross-sectional survey based on self-completed purpose-designed postal questionnaires.

\section{Sample}

The National Association of Paediatric Occupational Therapists (NAPOT) in the UK was used as a sampling frame. One hundred out of 300 occupational therapists were randomly chosen from the list provided. This size was considered as manageable in terms of time and resources to access and analyse the data obtained. Out of the 100 questionnaires sent, 51 were returned. Nine of them were not completed, as the therapists did not have enough knowledge or experience with the children under investigation. Forty-two completed questionnaires were used for the analysis, giving an effective response rate of $42 \%$, which is considered acceptable for postal surveys (De Vaus 2002).

\section{Procedure}

A survey, including a covering letter, a copy of the ethical approval granted by Brunel University, the questionnaire and a reply paid envelope, was mailed to the selected potential informants. The covering letter emphasised that the questionnaire should only be completed by therapists with clinical experience (current or past) with children who had both ADHD and LD either in reading, spelling, mathematics, or written expression with at least average intelligence. The covering letter also outlined the purpose of the study, the voluntary nature of participation, assured anonymity and confidentiality, and requested the return of the questionnaires using the self-addressed prepaid envelopes. Reminder letters along with the covering letter, the ethical approval, the questionnaire and the prepaid envelope were sent a month after the first contact to maximise the response rate. 


\section{Instrument}

The questionnaire included both closed and open questions. One item asked respondents whether they had worked with children presenting both ADHD and LD. A second item elicited information about therapists' self-assessed competence in the rehabilitation of these children. A third item explored the use of intervention methods and approaches by therapists in managing the targeted children. A fourth item requested the rationale underpinning the use of their preferred methods. The respondents had to tick boxes to indicate their preferred choices for closed questions. Spaces for further comments and for open questions were also provided. The questionnaire included further items, which are not discussed here as they explored issues which do not constitute the focus of this paper.

The questionnaire was pilot tested with five paediatric occupational therapists known to the author to improve content validity, clarity of the questionnaire and wording (Robson 2002). Honesty was pursued by ensuring anonymity of the responses. Depth was achieved by the inclusion of the option 'other' and openended questions which allowed exploration of the responses in more detail.

\section{Data Analysis}

Both quantitative and qualitative analysis was used. The responses from closed questions was analysed quantitatively. Descriptive statistics (frequencies and percentages) were used to summarise the information provided where a 'yes-nodo not know' format had been used. Confidence interval $(\mathrm{Cl})$ analysis based on the sample proportions were used to infer information regarding the proportion of the therapists in the population who use each method (Domholdt 2005). A process of thematic analysis was used for the open-ended questions. Closely linked concepts with similar meanings were identified and grouped together into sentences that represented therapists' views (Holloway 2007).

\section{Results}

\section{Therapists' Experience and Competence with Children with ADHD and LD}

Participants were asked whether they had worked with children presenting both ADHD and LD. They had to choose between 'yes', 'no', 'do not know'. The results confirmed that all respondents had working experience with these children.

Therapists were asked to comment on their perceived competency in the rehabilitation of these children. They had to choose between 'not competent', 'competent', 'higher than average competent' and 'expert'. The majority of therapist $(\mathrm{N}=34$ ) classified themselves as 'competent', six viewed themselves as possessing 'higher than average competence', whereas two were self-classified as 'experts in the field'.

\section{Methods and Approaches}

Participants were requested to indicate the approaches they used in their practice with children with ADHD and LD. Respondents had to choose from a list but also had the option of 'other', if their preferred approach was not included in the list. They were allowed to choose more than one method and the results showed that therapists typically used more than one $(N=38)$. All the listed approaches 
appeared to be quite common (as they were used by more than half of respondents), with the exception of 'computers' which were used by approximately a third of informants $(N=15)$. Sensory integration appeared as the most popular (used by 37 of 42 participants), followed by perceptual-motor training ( $N=32$ ). Therapeutic recreation, cognitive and behavioural approaches were used almost equally ( $N=23, N=22, N=22$, respectively) (table 1 ).

The results are reported in number of responses $(\mathrm{N})$ and percentages (\%). Total number of respondents $=42$

Table 1: The interventions used by therapists participating in the survey in the rehabilitation of children with ADHD and LD.

\begin{tabular}{lllll}
\hline & $\begin{array}{l}\text { Number of } \\
\text { responses }\end{array}$ & Percentage & 95\% Cl for $\mathbf{\pi}$ \\
& $\mathbf{N}$ & $\%$ & Lower limit & Upper limit \\
\hline $\begin{array}{l}\text { Sensory Integration } \\
\text { Approach }\end{array}$ & 37 & 88 & 78 & 98 \\
\hline Behavioural Approach & 22 & 52 & 37 & 67 \\
\hline Cognitive Approach & 22 & 52 & 37 & 67 \\
\hline Perceptual-Motor Training & 32 & 76 & 63 & 89 \\
\hline Computers & 15 & 36 & 21 & 51 \\
\hline Therapeutic Recreation & 23 & 55 & 40 & 70 \\
\hline Other & 12 & 29 & 16 & 43 \\
\hline Note: Cl: confidence & & & & \\
\hline
\end{tabular}

Note: $\mathrm{Cl}$ : confidence intervals, $\pi=$ true but unknown population

\section{Proportion}

Other methods were introduced by 12 therapists. These therapists suggested an 'eclectic approach', 'sensory stimulation', 'educational approach to teachers regarding sensory processing considerations', 'environmental adaptation', 'music therapy', 'group therapy', 'family therapy', and 'community training'. Therapists also suggested activities related to 'therapeutic recreation'. These involved active movement, such as physical play and sports, and activities based on the sensory integration principles.

Confidence interval analysis suggested that, with a 5\% probability of error, sensory integration is used by a higher percentage of occupational therapists. This is indicated by the results that the calculated lower limit $(78 \%)$ of the sensory integration's confidence interval exceeds the upper limit of the confidence intervals of the remaining approaches. The only exception is perceptual motor training, as its confidence interval $(63 \%-89 \%)$ overlaps with the one of sensory integration $(78 \%-98 \%)$. Hence, there is no robust statistical evidence to assert that sensory integration is more popular than perceptual motor training. Furthermore, one cannot reject the hypothesis that the cognitive, behavioural, therapeutic recreation, the 'other' approaches and the computers are equally popular among occupational therapists, as their confidence intervals overlap (table 1).

Participants were asked to provide the rationale for using their preferred methods. The main emerging themes were: 
1. The sensory integration approach was thought to be more successful for use with attention and educational difficulties coupled with underlying sensorimotor dysfunctions $(\mathrm{N}=11)$. The provision and control of sensory input was deemed to enable the integration of adaptive responses, and to facilitate the modulation of arousal leading to increased attention and successful academic work $(\mathrm{N}=13)$. Therapists also suggested that the sensory integration approach enables the development of social skills and improves peer-relationships and self-confidence $(\mathrm{N}=9)$. Additional positive effects were that it was fun and motivating for the child $(\mathrm{N}=4)$. Some therapists suggested that sensory integration has both immediate and longer lasting effects $(\mathrm{N}=3)$. Other therapists considered sensory integration to be particularly effective when applied as a long lasting on-going programme and when its principles are incorporated in the daily life of the child at home and at school $(\mathrm{N}=11)$.

2. Therapists reported that they use the perceptual motor training approach when they aim to treat the underlying causes of a child's problem $(\mathrm{N}=6)$. They suggested that it enables the development of functional skills that incorporate aspects of attention related to perceptual-motor skills $(\mathrm{N}=11)$. Also, perceptual motor training was considered by therapists to improve the components of movement needed for appropriate classroom behaviour (i.e. posture, shoulder stability, pencil control, in-hand manipulation, hand-eye co-ordination, sitting posture, visual perceptual and visual motor needs) $(\mathrm{N}=5)$.

3. Therapists suggested that they use therapeutic recreation mainly to increase function through fun play, social activities and learning from peers $(\mathrm{N}=9)$. They reported using this approach when they wish to address emotional issues related to a child $(\mathrm{N}=7)$. They also incorporate multi-sensory activities into therapeutic recreation to promote a high level of cognitive and work output skills and to improve sensori-motor abilities (N 7).

4. The behavioural approach seemed to be preferred by occupational therapists due to its focus on clear structures and boundaries to limit distractibility and organise behaviour $(\mathrm{N}=8)$. Moreover, its principal aspects of practice and repetition were thought to change maladaptive malfunctioning behaviour to adaptive behaviour $(\mathrm{N}=7)$. Therapists also reported that the behaviouristic approach enables the building of appropriate behaviour and sense of responsibility along with consequences of actions $(\mathrm{N}=7)$.

5. Therapists reported using the cognitive approach due to its focus on structuring the environment and providing context coding. They believed that this enables the child to carry out tasks $(\mathrm{N}=11)$. The cognitive method was also thought to enable self-awareness and to enhance self-monitoring, which will consequently lead to maintenance of appropriate behaviours or to the improvement of inappropriate behaviours $(\mathrm{N}=11)$.

6. Computers were mainly suggested by occupational therapists as an alternative method to motivate children. This motivation is thought to be achieved through typing and clicker programmes that can help with spelling issues $(\mathrm{N}=8)$. Computers were also used by therapists to enable visual training and control over the environment $(\mathrm{N}=7)$.

7. An interesting point emerging from the occupational therapists who suggested 'other approaches' was that they emphasised the need for an eclectic 
approach. They stated that an eclectic approach will better meet the specific goals for each child. They based this suggestion on the notion that no single treatment has been proven to work any better than the others and each child has different needs $(\mathrm{N}=2)$. Among the 'other' suggested approaches was 'sensory stimulation', which is believed by occupational therapists to improve a child's engagement and exploration of objects and their environment $(\mathrm{N}=2)$. Also, 'sensory processing features' were suggested as helpful to improve attention control through decrease of defensiveness and enhancement of sensory modulation $(\mathrm{N}=2)$. Therapists also introduced 'environmental adaptation' based on the premise that suitable environments will enable children to focus their attention $(\mathrm{N}=2)$.

\section{Discussion}

\section{Intervention with Children with ADHD/LD}

An important finding of this study is that paediatric occupational therapists tend to integrate more than one approach in their intervention with children who appear with both ADHD and LD. Two therapists specifically introduced this as a separate entry at the section of 'others' referring to it as 'eclectic approach'. This is not surprising when one considers that children with this overlap might also present with other disorders. It has been suggested that over $50 \%$ of ADHD children may be influenced by one or more of the associated co-morbidities (Brown 2000), therefore it is unsurprising that such complex impairment as these require a multimodal/multi-faceted approach (Cavanaugh et al 1997, Foy \& Earls 2005). The effectiveness of such a programme has been piloted by $\mathrm{Chu}$ and Reynolds (2007b) on 20 children with ADHD indicating positive results. A valuable contribution would be a well designed effectiveness study to assess the effects of multiple interventions on children who appear with both ADHD and LD compared with single approaches. This would allow the cumulative benefits of multiple approaches to be estimated and whether together they have greater impact than single interventions.

Therapists suggested that no method has been proven superior to others. This statement may reflect the absence of evidence-based practice in this area, and/or the difficulties in assessing the effectiveness of specific methods with such complex impairments. Still, sensory integration was indicated as the most preferred method by the sample occupational therapists. The above, in combination with the results of Howard's (2002) national survey with UK paediatric occupational therapists, which revealed that the sensory integration training was the most commonly accessed course in UK, raises questions worthy of further consideration. The therapists who participated in this study may have indicated a preference for sensory integration on the basis they had undertaken relevant training and therefore felt more confident, or they might have had evidence from observing positive clinical results with this subgroup of children. Indeed, techniques related to the sensory integration approach have been shown to be effective for managing ADHD. VandenBerg (2001) showed an increase in functional attention during purposeful activity when deep pressure sensory input was applied through the use of a weighted vest in ADHD children in a classroom environment. Schilling et al (2003) showed improvement in classroom behaviour when children with ADHD were seated on a therapy ball, which acted as a source of vestibular and proprioceptive stimulation. However, studies on the effectiveness of sensory integration approaches with children with both ADHD and LD were not identified. 
The second preferred method reported by the sample occupational therapists was perceptual motor training. Indeed, a perceptual motor training programme in computer-format named 'the interactive metronome' was found to be successful in improving complex problem solving behaviours in school, at home and in social relationships in children with ADHD (Koomar et al 2001). However, its effectiveness remains to be shown with children who appear with both ADHD and LD. No other studies were identified that demonstrated the effectiveness of other perceptual motor training techniques with these children.

Cognitive and behavioural methods, and therapeutic recreation were equally chosen by occupational therapists. Cognitive strategies (i.e. teaching the use of self-instruction, self-monitoring and self-reinforcement) have been found useful in the amelioration of executive dysfunction associated with the symptoms of inattention (Graham \& Harris 1996). A cognitively-based approach, such as the 'alert programme' (Williams \& Shellenberger 1994) has been used to assist parents and children in monitoring, maintaining and changing level of alertness. Behavioural therapy has also been considered effective for ADHD (Herbert 1994), particularly if carried out with parents' cooperation (Hornby et al 1997). Studies have shown that behavioural interventions (i.e. positive reinforcement, punishment and response cost) in combination with stimulant medication can be more effective than medication by itself (Pelham et al 1993). Interestingly, Ervin et al (1996) suggested the combination of cognitive with behavioural contingencies in children's natural environment to increase motivation and possibly enhance effect for children with ADHD. Still, effectiveness studies need to be carried out with children with both ADHD and LD.

Therapists linked the use of therapeutic recreation (i.e. active play and sports) to its emotional and social benefits that consequently impact on children's improved function. Indeed, therapeutic recreation has been found as beneficial in physical, cognitive and social domains. Exercise play is thought to heighten arousal (Pellegrini \& Smith 1998) and to provide children with ADHD the chance to 'run off' excess energy (Fanchiang 1996). Still, Fanchiang (1996) suggested that the benefits of therapeutic recreation have been assumed, rather than studied in their own rights. This would also apply in the effectiveness of such occupations with children with both ADHD and LD.

Finally, computers were less favoured by the sample occupational therapists. Interestingly, those who did use them linked them either with the cognitive approach or with environmental adaptations. Still, the 'interactive metronome' is a computer programme based on the perceptual motor training approach. Also, computer-based auditory programmes are being tested in children with ADHD and dyslexia (Shaywitz 1996) for improvement of language and reading problems. Unsworth and Townsend (1997) suggested that therapists should be knowledgeable and skilled in technology in order to use it for their clients' advantage. The fact that computers were less popular in the sample occupational therapists might be related to lack of relevant knowledge or resources. Indeed, studies in the US reported that occupational therapists may not be using adequate computers (Spicer \& McMillan 1987, Somerville et al 1990; Weiss 1990). Some of the reported reasons were related to the negative attitude of occupational therapists towards computers (Weiss 1990), the lack of relevant education and experience (Weiss 1990) and the lack of resources (Spicer \& McMillan 1987). 
Interestingly, the occupational therapists in this study reported introducing methods such as music therapy and family therapy, which are traditionally associated with other disciplines. Also, methods such as the behavioural and the cognitive approach are being used by other professionals as well, in the rehabilitation of children with ADHD. This indicates the importance of collaborative and integrated intervention in which two or more professionals will bring together resources to meet objectives that neither could meet individually (Graham \& Barter 1999). It also indicates that collaborative teamwork and communication among different disciplines is a necessity for efficient use of resources and for planning and coordination of services. Finally, the absence of studies specifically examining the rehabilitation of children who experience both ADHD and LD suggests the necessity for future studies to test the effectiveness of different methods with these children using a multidisciplinary approach.

\section{Limitation of the Study}

Thirty-four out of 42 therapists viewed themselves as 'just competent' in supporting children with both ADHD and LD. A greater response from experts in the field might have provided further information regarding best practice in the rehabilitation of these children. The instrument did not elicit demographic information about the education, training or volume of experience with children with ADHD/LD. This information would have enabled more detailed interpretation of the results. Using the NAPOT database for sampling increased the potential of recruiting a study sample with more specialised knowledge in paediatrics. However, therapists working with the targeted children who were not NAPOT members were excluded and this inevitably impacts on the generalisability of the results. Therapists who had worked in the past but were not working at the time of the study with these children, were also invited to respond. This might have introduced bias, as therapists had to rely on their memory to complete the questionnaire. It should also be noted that each child is a case with idiosyncratic characteristics - a fact that is largely ignored when questions refer to groups of clients, as in this study. Due to all the above and the small-sample bias these findings should be treated as indicative, calling for further research in this field.

\section{Conclusion}

This small-scale survey examined issues concerning current practice of UK paediatric occupational therapists involved in the management of children with both $A D H D$ and LD. Findings suggested that techniques related to sensory integration and perceptual motor training were most frequently used by occupational therapists with this group of children. Still, a variety of other approaches, which could be used by other professionals as well as occupational therapists, were suggested as having a place when providing a needs based individualised programme of care. These results suggest that effective multidisciplinary collaboration, recognising the occupational therapy contribution, is significant in addressing the holistic needs of these children. The reported intervention approaches could be used for future studies using single case experimental designs where these interventions could be investigated as to whether they are effective for the targeted children and under what circumstances. Moreover, further research is needed to establish how these methods can be incorporated into effective multidisciplinary working in order to meet the complex needs of these children. 
Mgmt of Children with Attention Deficit/Hyperactivity Disorder \& LD:

A Survey of Paediatric Occupational Therapists in the UK

\section{Acknowledgements}

The author wishes to thank NAPOT and the occupational therapists who have participated in the study. Acknowledgements are also given to IKY-Greek State Scholarship's Foundation in awarding the scholarship for the author's postgraduate studies, under which this project has been completed. 


\section{References}

American Psychiatric Association 1994. Diagnostic and Statistical Manual of Mental Disorders (DSM - IV). $4^{\text {th }}$ ed. Washington, D.C, American Psychiatric Press.

Ayres, A.J. 1979. Sensory Integration and the Child. Los Angeles, Western Psychological Services.

Barkley, R.A. 1994. What to Look for in a School for a Child with ADHD. ADHD Report 2(3): 1-3.

Barkley, R.A. 2000. Genetics of Childhood Disorders: XVII. ADHD, part 1: The Executive Functions and ADHD. Journal of the American Academy of Child and Adolescent Psychiatry 39: 1064-68.

Biederman, J., Newcorn, J. \& Sprich, S. 1991. Comorbidity of Attention Deficit with Conduct, Depressive, Anxiety and Other Disorders. American Journal of Psychiatry 148: 564-77.

Brown, T.E. 2000. Attention-Deficit Disorders and Comorbidities in Children, Adolescents, and Adults. Washington, DC, American Psychiatric Press.

Cassily, J.F. 1996. Methods and Apparatus for Measuring and Enhancing Neural Motor Coordination. United States Patent 5: (529): 498.

Castellanos, X.F. 1997. Toward a Pathophysiology of AttentionDeficit/Hyperactivity Disorder. Clinical Pediatrics: 381-93.

Cavanaugh, S., Tervo, R.C., \& Fogas, B. 1997. The Child with Attention Deficit Hyperactivity Disorder and Learning Disability. South Dakota Journal of Medicine 50(6): 193-97.

Chu, S. 2003. Occupational Therapy for Children with Attention Deficit Hyperactivity Disorder: A Survey on the Level of Involvement and Training Needs of Therapists. British Journal of Occupational Therapy 66(5): 209-18.

Chu, S., \& Reynolds, F. 2007a. Occupational Therapy for Children with Attention Deficit Hyperactivity Disorder (ADHD), part 1: A Delineation Model of Practice. British Journal of Occupational Therapy, 70(9): 372-383.

Chu, S., \& Reynolds, F. 2007b. Occupational Therapy for Children with Attention Deficit Hyperactivity Disorder (ADHD), Part 2: A Multicentre Evaluation of an Assessment and Treatment Package. British Journal of Occupational Therapy 70(10): 439-448.

Department of Health 2001. Valuing people: A New Strategy for Learning Disability for the $21^{\text {st }}$ Century. London, HMSO.

DeVaus, D. 2002. Surveys in Social Research. $5^{\text {th }}$ ed. London, Routledge.

Domholdt, E. 2005. Rehabilitation Research: Principles and Applications. $3^{\text {rd }}$ ed. St. Louis, Elsevier Saunders. 
Dunn, W. 1999. Sensory Profile: Examiner's Manual. San Antonio, TX, Psychological Corporation.

Ervin, R.A., Bankert, C.L., \& DuPaul, G.J. 1996. Treatment of attentionDeficit/Hyperactivity Disorder. In Reinecke, M.A., \& Dattilio, F.M. Cognitive therapy with Children and Adolescents: A Casebook for Clinical Practice, New York, Guilford Press: 38-61.

Fanchiang, S.C. 1996. The Other Side of the Coin: Growing Up with a Learning Disability. The American Journal of Occupational Therapy 50(4): 277-284.

Foy, J.M., \& Earls, M.F. 2005. A Process for Developing Community Consensus Regarding the Diagnosis and Management Of Attention-Deficit/Hyperactivity Disorder. Pediatrics 115(1): 97-104.

Graham, S., \& Harris, K.R. 1996. Addressing Problems in Attention, Memory, and Executive Functioning. An Example from Self-Regulated Strategy Development. In Lyon, G.R., \& Krasnegor, N.A. Attention, Memory, and Executive Function. Baltimore, Paul H Brookes Publishing Co: 349-65.

Grizenko, N., Bhat, M., Swartz, G., Ter-Stephanian, M., \& Joober, R. 2006. Efficacy of Methylphenidate in Children with Attention-Deficit Hyperactivity Disorder and Learning Disabilities: A Randomized Crossover Trial. Journal of Psychiatry and Neuroscience 31(1): 46-51.

Herbert, M. 1994. Behavioural Methods. In Rutter, M., Taylor, E., \& Hersov, L. Child and Adolescent Psychiatry: Modern Approaches. $3^{\text {rd }}$ ed. Oxford, Blackwell Scientific.

Hornby, G., Atkinson, M., \& Howard, J. 1997. Controversial Issues in Special Education. London, David Fulton Publishers.

Holloway, I. 1997. Basic concepts for qualitative research. Melbourne, Blackwell Science.

Howard, L. 2002. A survey of Paediatric Occupational Therapists in the United Kingdom. Occupational Therapy International 9(4): 326-43.

Kaplan, B.J., Dewey, D.M., Crawford, S.G., \& Wilson, B.N. 2001. The Term CoMorbidity is of Questionable Value in Reference to Developmental Disorders: Data and Theory. Journal of Learning Disabilities 34: 555-565.

Koomar, J., Burpee, J.D., DeJean, V., Frick, S., Kawar, M.J., \& Fisher, D.M. 2001. Theoretical and clinical perspectives on the interactive metronome: A View from Occupational Therapy Practice. American Journal of Occupational Therapy 55(2): 163-66.

Lazar, J.W., \& Frank, Y. 1998. Frontal Systems Dysfunction in Children with Attention Deficit/Hyperactivity Disorder and Learning Disabilities. The Journal of Neuropsychiatry and Clinical Neurosciences 10: 160-67.

Mangeot, S.D., Miller, L.J., McIntosh, D.N., McGrath-Clarke, J., Simon, J., Hagerman, R.J., \& Goldson, E. 2001. Sensory Modulation Dysfunction in Children with Attention-Deficit-Hyperactivity Disorder. Developmental Medicine and Child Neurology 43: 399-406. 
Mayes, S.D., Calhoun, S.L., \& Crowell, E.W. 2000. Learning Disabilities and ADHD: Overlapping Spectrum Disorders. Journal of Learning Disabilities 33(5): 417-24.

National Institute for Clinical Excellence 2000. Technology Appraisal Guidance No.13 - Guidance on the Use Of Methylphenidate (Ritalin, Equasym) for Attention Deficit Hyperactivity Disorder (ADHD) In Childhood. London, (National Institute of Clinical Excellence (NICE).

National Joint Committee on Learning Disabilities 2001. Learning disabilities: Issues on definition. In National Joint Committee on Learning Disabilities. Collective Perspectives on Issues Affecting Learning Disabilities: Position papers, statements, and reports. $2^{\text {nd }}$ ed. Austin, TX, Pro-Ed: 27-32.

Oetter, P. 1986. A Sensory Integrative Approach to the Treatment of Attention Deficit Disorders. Sensory Integration Special Interest Section Newsletter 9(2): 12.

Parush, S., Sohmer, H., Steinberg, A., \& Kaitz, M. 1997. Somatosensory Functioning in Children with Attention Deficit Hyperactivity Disorder. Developmental Medicine and Child Psychology 39(7): 464-68.

Pelham, W.E., Carlson, C.L., Sams, S.E., \& Vallano, G. 1993. Separate and Combined Effects of Methylphenidate and Behaviour Modification on Boys with Attention Deficit-Hyperactivity Disorder in the Classroom. Journal of Consulting and Clinical Psychology 61: 506-15.

Pellegrini, A.D., \& Smith, P.K. 1998. Physical Activity Play. Child Development 69(3): 577-598.

Riccio, C., Hynd, G., Cohen, M., \& Gonzalez, J. 1993. Neurological Basis of Attention Deficit Hyperactivity Disorder. Exceptional Children 60(2): 118-24.

Robson, C. 2002. Real world research. $2^{\text {nd }}$ ed. UK, Blackwell Publishing.

Rosenman, S. 2006. Reconsidering the Attention Deficit Paradigm. Australasian Psychiatry 14(2): 127-32.

Schilling, D.L., Washington, K., Billingsley, F.F., \& Deitz, J. 2003. Classroom Seating for Children with Attention deficit Hyperactivity Disorder: Therapy Balls Versus Chairs. American Journal of Occupational Therapy 57(5): 534-41.

Shaywitz, S.E. 1996. Dyslexia, Scientific American, November: 98-104.

Somerville, N., Wilson, D., \& Shanfield, K. 1990. A Survey of the Assistive Technology Training Needs of Occupational Therapists. Assistive Technology 2: 41-49.

Spicer, M., \& McMillan, S. 1987. Computers and Occupational Therapy. American Journal of Occupational Therapy 41: 726-32.

Spreen, O. 2001. Learning Disabilities and their Neurological Foundations, Theories, and Subtypes. In Kaufman, A.S., \& Kaufman, N.L. Specific Learning Disabilities and Difficulties in Children and Adolescents: Psychological Assessment and evaluation. Cambridge University Press: 283-308. 
Unsworth, C.A., \& Townsend, M.C. 1997. Occupational Therapists' Skills and Attitudes Regarding use of Computers and Assistive Technology. Occupational Therapy International 4(1): 52-65.

VandenBerg, N.L. 2001. The Use of a Weighted Vest to Increase On-Task Behaviour in Children With Attention Difficulties. American Journal of Occupational Therapy 55(6): 621-8.

Weiss, P. 1990. The Integration of Computers into the Occupational Therapy Department. American Journal of Occupational Therapy 44: 527-34.

Whitaker, S. 2006. What's in a Name? Alternatives to 'Learning Disability'. Mental Health and Learning Disabilities: Research and Practice 3(2): 178-191.

Williams, M.S., \& Shellenberger, S. 1994. How Does Your Engine Run? A Leader's Guide to the Alert Program for Self-Regulation. Albuquerque, NM, Therapy-Works. 